\title{
Nutritional effects on auditory brainstem maturation in healthy term infants
}

\author{
B Ünay, S Ü Sarıcı, Ü H Ulaș, R Akın, F Alpay, E Gökçay
}

Arch Dis Child Fetal Neonatal Ed 2004;89:F177-F179. doi: 10.1136/adc.2002.021014

\begin{abstract}
Objective: To assess the effects of dietary long chain polyunsaturated fatty acid (LCPUFA) supplementation on auditory brainstem maturation of healthy term newborns during the first 16 weeks of life by measuring brainstem auditory evoked potentials (BAEPs).

Design: Throughout the 16 week study period, infants in the formula $A$ group ( $n=28$ ) were assigned to be fed exclusively with the same formula supplemented with DHA, and infants in the formula $B$ group $(n=26)$ were assigned to receive only a DHA unsupplemented but otherwise similar formula. During the study period, the first 26 consecutive infants to be fed exclusively on their mother's milk for at least the first 16 weeks of life were chosen as the control group. BAEP measurements were performed twice: at the first and 16 th week of age.

Results: There were no significant differences among the study and control groups in the BAEP measurements performed at the study entry. At 16 weeks of age, all absolute wave and interpeak latencies in the study and control groups had significantly decreased. The decreases were significantly greater in the formula $A$ and control groups than in the formula B group.

Conclusions: Infants fed on human milk or a formula supplemented with LCPUFAs during the first 16 weeks of life show more rapid BAEP maturation than infants fed on a standard formula. Although the clinical importance and long term effects of these findings remain to be determined, routine supplementation of formulas with LCPUFAs should be considered.
\end{abstract}

ong chain polyunsaturated fatty acids (LCPUFAs), especially arachidonic acid (20:4n-6) and docosahexaenoic Lacid (DHA; 22:6n-3), are essential for brain growth and cognitive development, and breast milk provides a good supply of arachidonic acid and DHA. Preterm infants fed with breast milk have been reported to show greater neuronal maturation than formula fed preterm infants on the basis of determination of visual evoked potentials and electroretinography. ${ }^{12}$ Furthermore, both preterm and term newborns fed with a formula supplemented with DHA show more rapid and better development of visual acuity and better psychomotor development than infants fed with a standard formula. ${ }^{2-4}$

The effect of breast feeding on the maturation of brainstem auditory evoked potentials (BAEPs) in exclusively breast fed preterm infants has been investigated and compared with formula fed preterm infants in two studies. ${ }^{56}$ However, the effects of dietary LCPUFA supplementation on brainstem maturation during early infancy has, to our knowledge, not previously been investigated in term newborns. In this study we aimed to assess the effects of dietary LCPUFA supplementation on auditory brainstem maturation of healthy term newborns during the first 16 weeks of life by measuring BAEPs.

\section{METHODS}

The study was performed on infants born at the Obstetrics Clinics of Gülhane Military Medical Academy between November 2000 and September 2001 and regularly followed up at the well baby clinics of the same hospital. The study groups consisted of 54 healthy, full term newborns of appropriate size for gestational age, who were not going to be breast fed because that was the mother's wish or because of maternal illness or medication incompatible with breast feeding just after birth. These newborns were randomly allocated using a computer based software program to one of the study (formula) groups in a double blind fashion within the first week of life. Throughout the study period of 16 weeks, infants in the formula A group $(n=28)$ were assigned to be fed exclusively with the same formula supplemented with DHA (formula A; Farley's First Milk, HJ Heinz Co, UK), and the infants in the formula B group ( $\mathrm{n}=26$ ) were assigned to receive only a DHA unsupplemented but otherwise similar formula (formula B; Nutrilon I; NV Nutricia, Netherlands). During the study period, the first 26 consecutive infants who were to be fed exclusively on their mother's milk for at least the first 16 weeks of life were chosen as the control group.

Newborns with perinatal asphyxia, central nervous system infection, congenital malformation, or significant hyperbilirubinaemia were excluded from the study. Parents were requested to strictly adhere to the assigned feeding protocol (formula A or B, or breast milk) and not to introduce any solid or additional foods before 16 weeks of age. The formulas were prepared by someone other than the investigators ( $\mathrm{N}$ Inanc), and the formula packages concealed with wrapping paper. These formulas were provided to the parents in the formula receiving groups without the contents being revealed. Whether and how the parents conformed with the feeding protocol was monitored by two of the investigators (BU and SUS) on a weekly basis until completion of the study.

BAEP measurements were made twice; at the first and 16 th week of age. The potentials were recorded as described in detail elsewhere. ${ }^{7}$ Absolute latencies for waves I, III, and V and interpeak latencies I-III, III-V, and I-V were recorded. Latency values obtained for left and right ears were averaged to represent one value in each case. Decreases in absolute wave and interpeak latencies were calculated by subtracting the first measurement from the second for each.

Abbreviations: BAEP, brainstem auditory evoked potential; DHA docosahexaenoic acid; LCPUFA, long chain polyunsaturated fatty acid 
Table 1 Fatty acid compositions ( $\mathrm{g} / 100 \mathrm{~g}$ fat) of the two formulas used in the study and human milk

\begin{tabular}{llll}
\hline Fatty acid & $\begin{array}{l}\text { Formula } \\
\text { A }\end{array}$ & $\begin{array}{l}\text { Formula } \\
\text { B }\end{array}$ & $\begin{array}{l}\text { Human } \\
\text { milk }\end{array}$ \\
\hline Myristic acid (14:0) & 4.2 & 4.5 & 6.27 \\
Palmitic acid (16:0) & 14 & 18.1 & 22 \\
Stearic acid (18:0) & 3.7 & 3.9 & 8.06 \\
Oleic acid (18:1n-9) & 50.8 & 44.9 & 31.3 \\
Linoleic acid (18:2n-6) & 9.7 & 11.2 & 10.85 \\
$\begin{array}{l}\alpha \text {-Linoleic acid (18:3n-3) } \\
\text { Arachidonic acid }\end{array}$ & 1.2 & 2.2 & 1.03 \\
(20:4n-6) & & - & 0.46 \\
Docosahexaenoic acid & 0.5 & - & 0.25 \\
(22:6n-3) & & & \\
\hline
\end{tabular}

Formula A, Farley's First Milk; formula B, Nutrilon I.

Table 2 Brainstem auditory evoked potentials of the study and control groups performed at entry into the study

\begin{tabular}{lllll}
\hline & $\begin{array}{l}\text { Formula A } \\
(\mathbf{n = 2 2 )}\end{array}$ & $\begin{array}{l}\text { Formula B } \\
(\mathbf{n}=\mathbf{2 2})\end{array}$ & Control $(\mathbf{n = 2 3 )} \mathbf{p}$ Value \\
\hline Wave I & $2.8(0.17)$ & $2.86(0.21)$ & $2.8(0.2)$ & $>0.05$ \\
Wave III & $5.64(0.32)$ & $5.68(0.27)$ & $5.65(0.21)$ & $>0.05$ \\
Wave V & $7.9(0.23)$ & $7.94(0.22)$ & $7.96(0.28)$ & $>0.05$ \\
IPL I-III & $2.84(0.22)$ & $2.82(0.22)$ & $2.85(0.22)$ & $>0.05$ \\
IPL III-V & $2.26(0.32)$ & $2.26(0.25)$ & $2.31(0.32)$ & $>0.05$ \\
IPL I-V & $5.1(0.24)$ & $5.08(0.28)$ & $5.16(0.25)$ & $>0.05$ \\
\hline
\end{tabular}

Values (milliseconds) are given as mean (SD).

Formula A, Farley's First Milk; formula B, Nutrilon I; IPL, interpeak latency.

Written informed parental consent was obtained for each infant, and the study was approved by the local ethics committee.

Basic characteristics, absolute wave latencies, and interpeak latencies of the three groups in the study were compared using analysis of variance with post hoc multiple comparison of Tukey or Tamhane tests.

\section{RESULTS}

Eighty newborns were initially included in the study. However, only 44 in the study groups (22 in each group) and 23 in the control group completed the study because some of the parents did not want to continue participating and some of the subjects were lost during follow up. There were no significant differences among the three groups with respect to birth weight, gestational age, sex, and parameters of growth (weight, length, and head circumference) either at entry into or after completion of the study.

Fatty acid compositions of the human milk and two formulas used in the study were analysed by gas chromatography to confirm their LCPUFA content (table 1).

There were no significant differences among the study and control groups with respect to the BAEP measurements performed at the study entry (table 2). At 16 weeks of age, all absolute wave and interpeak latencies in the study and control groups had significantly decreased (fig 1). The decreases were significantly greater in the formula A and control groups than the formula B group $(\mathrm{p}<0.05)$ (table 3$)$.

\section{DISCUSSION}

Both preterm ${ }^{16}$ and term ${ }^{8}$ newborn infants fed with a formula unsupplemented with LCPUFAs have been shown to have lower plasma, tissue, and erythrocyte concentrations of LCPUFAs than infants fed with human milk. This must be due to the large amount of LCPUFAs present in human milk, as most of the standard formulas are fortified only with precursor essential fatty acids such as $\alpha$-linolenic acid (18: $3 n-3)$ and linoleic acid (18: 2n-6).

In one of the two studies that have examined the effect of type of enteral feeding on the maturation of auditory functions in preterm infants, Amin et al showed a significantly increased maturation rate of BAEPs in 17 exclusively breast fed infants compared with 20 formula fed infants. They suggested that this effect may be attributable to the composition of breast milk, compared with synthetic formulas. In the other study of the influence of dietary LCPUFA supplementation on evoked potential maturation in 58 healthy preterm infants at 52 weeks of postconceptional age, Faldella et $a l^{6}$ showed a slower maturation pattern of
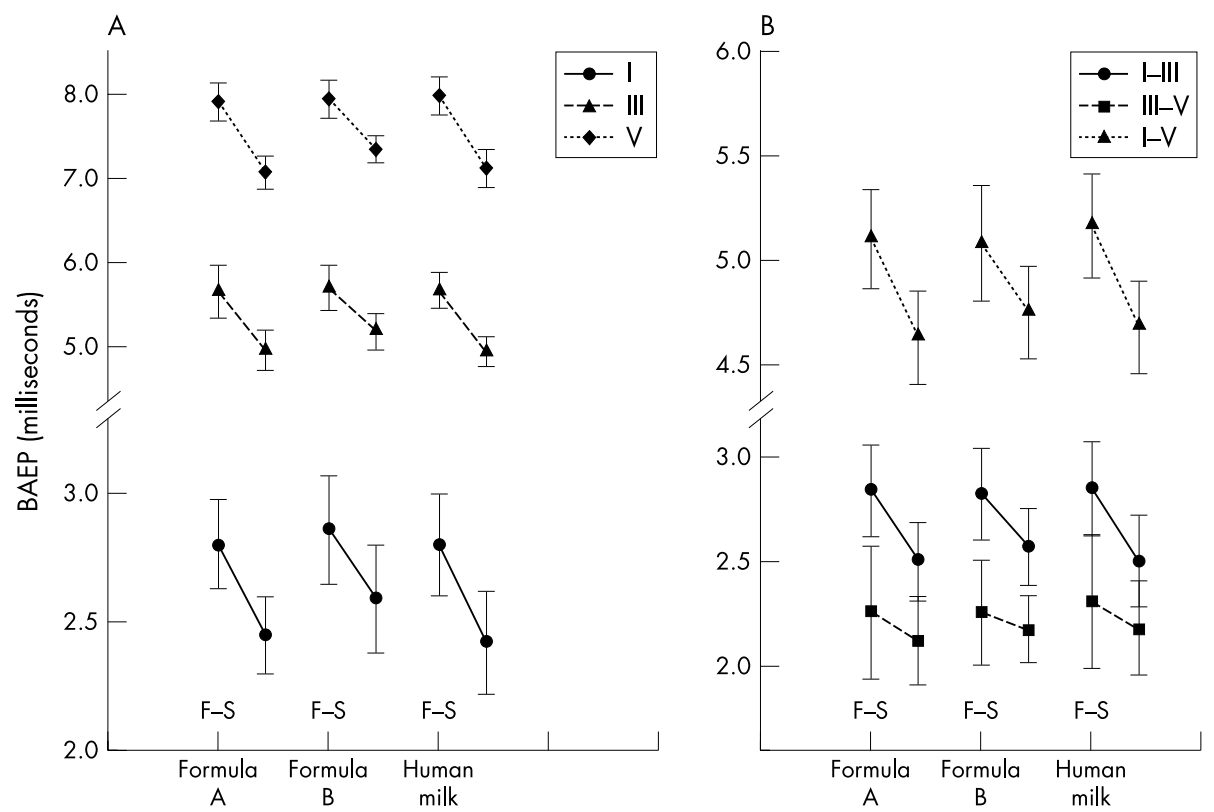

Figure 1 Changes in absolute wave (A) and interpeak (B) latencies in the study and control groups at 16 weeks of age (S, second) compared with the values obtained at study entry ( $F$, first). Formula A, Farley's First Milk; formula B, Nutrilon I 
Table 3 Decreases in absolute wave and interpeak latencies at 16 weeks of age*

\begin{tabular}{|c|c|c|c|c|}
\hline & $\begin{array}{l}\text { Formula A } \\
(n=22)\end{array}$ & $\begin{array}{l}\text { Formula B } \\
(n=22)\end{array}$ & $\begin{array}{l}\text { Control (C) } \\
(n=23)\end{array}$ & p Value* \\
\hline Wave I & $0.35(0.13)$ & $0.27(0.14)$ & $0.38(0.2)$ & $\begin{array}{l}A \vee B=0.03 \\
A \vee C=0.9 \\
B \vee C=0.02\end{array}$ \\
\hline Wave III & $0.69(0.16)$ & $0.52(0.15)$ & $0.73(0.15)$ & $\begin{array}{l}A \vee B=0.001 \\
A \vee C=0.53 \\
B \vee C=0.001\end{array}$ \\
\hline Wave V & $0.83(0.18)$ & $0.6(0.11)$ & $0.86(0.18)$ & $\begin{array}{l}A \vee B=0.001 \\
A \vee C=0.87 \\
B \vee C=0.002\end{array}$ \\
\hline IPL I-III & $0.34(0.16)$ & $0.25(0.14)$ & $0.35(0.22)$ & $\begin{array}{l}A \vee B=0.038 \\
A \vee C=0.99 \\
B \vee C=0.02\end{array}$ \\
\hline IPL III-V & $0.14(0.1)$ & $0.08(0.07)$ & $0.13(0.11)$ & $\begin{array}{l}A \vee B=0.023 \\
A \vee C=0.809 \\
B \vee C=0.015\end{array}$ \\
\hline IPL I-V & $0.47(0.2)$ & $0.33(0.16)$ & $0.48(0.2)$ & $\begin{array}{l}A \vee B=0.026 \\
A \vee C=0.99 \\
B \vee C=0.04\end{array}$ \\
\hline
\end{tabular}

Values (milliseconds) are given as mean (SD).

Formula A, Farley's First Milk; formula B, Nutrilon I; IPL, interpeak

latency.

*The differences between the formula $B$ group and each of the other two (formula $A$ and control) groups were significant $(p<0.05)$.

visual evoked potentials in 25 preterm infants receiving a traditional preterm formula compared with 21 and 12 preterm infants fed with LCPUFA supplemented formula and breast milk respectively. However, the authors could not detect any differences in BAEP values among the three groups. In the first study to evaluate the effects of different formulas and breast feeding on the evolution of BAEPs in healthy term infants during the first 16 weeks of life, we show a more rapid maturation of the absolute wave and interpeak latencies of infants fed with either breast milk or a formula supplemented with DHA compared with infants fed with a standard formula. However, we cannot exclude the possible effects of other fatty acids such as palmitic acid and oleic acid, as there are differences, though minor, between the concentrations of these fatty acids among the two formulas and human milk in our study. According to the study of Makrides et al, ${ }^{1}$ visual evoked potential acuities of infants who were breast fed or fed LCPUFA supplemented formula were higher than those of infants fed unsupplemented formula, indicating that DHA is an essential nutrient in optimal visual neuronal maturation of term infants. Agostoni et $a l^{4}$ have shown that term infants fed with LCPUFA supplemented formula scored significantly higher on the Brunet-Lezine scale than term infants who received standard formula at 4 months of age. They claim that the neurodevelopmental outcome of the healthy term infants who received LCPUFA supplemented formula was better than that of those who did not receive LCPUFA supplementation. All these findings suggest that both term and preterm infants can benefit with respect to neuronal maturation and neurodevelopmental performance from being fed with LCPUFA supplemented formulas.

Our results indicate that infants fed on human milk or a formula supplemented with LCPUFAs during the first 16 weeks of life exhibit more rapid BAEP maturation than infants fed on a standard formula. Although the clinical importance and long term effects of these findings remain to be determined in further longitudinal and large scale studies, routine supplementation of formulas with LCPUFAs for formula fed term newborns should be considered.

\section{ACKNOWLEDGEMENTS}

We thank Miss Neriman Inanc for her efforts in following the nutritional status of the infants.

\section{Aưthors' affiliations}

B Ünay, S Ü Sarıcı, Ü H Ulaș, R Akın, F Alpay, E Gökçay, Department of Pediatrics, Gülhane Military Medical Academy, Ankara, Turkey

Correspondence to: Dr Ünay, Department of Pediatrics, Gülhane Military Medical Academy, Etlik-06018, Ankara, Turkey;

bunay@gata.edu.tr

Accepted 7 February 2003

\section{REFERENCES}

1 Makrides M, Neumann M, Simmer K, et al. Are long-chain polyunsaturated fatty acids essential nutrients in infancy? Lancet 1995;345:1463-8.

2 Martinez $M$. Tissue levels of polyunsaturated fatty acids during early human development. J Pediatr 1992;120:S129-38.

3 Carlson SE, Werkman SH, Rhodes PG, et al. Visual-acuity development in healthy preterm infants: effect of marine-oil supplementation. Am J Clin Nutr 1993:58:35-42.

4 Agostoni C, Trojan S, Bellu R, et al. Neurodevelopment quotient of healthy term infants at 4 months and feeding practice: the role of long-chain polyunsaturated fatty acids. Pediatr Res 1995;38:262-6.

5 Amin SB, Merle KS, Orlando MS, et al. Brainstem maturation in premature infants as a function of enteral feeding type. Pediatrics 2000;106:318-22.

6 Faldella G, Govoni M, Alessandroni R, et al. Visual evoked potentials and dietary long chain polyunsaturated fatty acids in preterm infants. Arch Dis Child Fetal Neonatal Ed 1996;75:F108-12.

7 Sarici SÜ, Serdar MA, Dündaröz MR, et al. Brainstem auditory-evoked potentials in iron-deficiency anemia. Pediatr Neurol 2001;24:205-8.

8 Ponder DL, Innis SM, Benson JD, et al. Docosahexaenoic acid status of term infants fed breast milk of infant formula containing soy oil or corn oil. Pediatr Res 1992;32:683-8. 